\title{
Synchronization properties of three delay-coupled semiconductor lasers
}

\author{
Raúl Vicente* \\ Department of Neurophysiology, Max-Planck Institute for Brain Research, Deutschordenstrasse 46, 60528 Frankfurt am Main, Germany \\ Ingo Fischer ${ }^{\dagger}$ \\ School of Engineering and Physical Sciences and Joint Research Institute for Integrated Systems, Heriot-Watt University, \\ Edinburgh EH14 4AS, United Kingdom \\ Claudio R. Mirasso \\ Insituto de Física Interdisciplinar y Sistemas Complejos (IFISC), CSIC-UIB Campus Universitat de les Illes Balears, \\ 07122 Palma de Mallorca, Spain
}

(Received 2 June 2008; published 2 December 2008)

\begin{abstract}
We present detailed numerical studies of the dynamics of three semiconductor lasers when interacting in a linear chain through the mutual injection of their optical fields. In particular, we focus on the synchronization properties of the coupling-induced dynamics and the role of the delay in the interaction between the lasers. The recently experimentally and numerically demonstrated zero-lag synchronization [Fischer et al., Phys. Rev. Lett. 97, 123902 (2006)] between the outer lasers in the chain is here further analyzed in detail along with a study of the robustness of this phenomenon. In addition, the propagation properties of perturbing pulses and of harmonic modulation are discussed.
\end{abstract}

DOI: 10.1103/PhysRevE.78.066202

PACS number(s): 05.45.Xt, 42.55.Px, 42.65.Sf

\section{INTRODUCTION}

Collective behavior of coupled nonlinear oscillators is a fascinating subject which has captured the attention of researchers in very different fields [1,2]. Among the most prominent examples of emergent behaviors in such complex systems, synchronization describes the process by which rhythms of interacting entities are adjusted when they are properly coupled $[2,3]$. Synchronization has been found in a variety of systems that can be described as multiple weakly interacting subsystems such as biological [4-6], chemical [7], physical [8-10], and social systems [11]. In most of the studies carried out so far, the interaction between systems has been considered instantaneous, i.e., the information is transferred from one system to the other without delay. Whether this assumption is valid or not depends mainly on the comparison between the internal time scale of the systems under consideration and the time it takes for the information to propagate from one system to the other. In many examples the internal time scale is much slower than the information propagation time and thus the assumption of instantaneous coupling is valid; an instantaneous coupling also facilitates the analytical or numerical study which otherwise is more complicated due to the inclusion of time delays in the modeling equations. However, in the field of optics there is a simple and particular example for which the assumption of instantaneous coupling fails: when coupling two or more semiconductor lasers (SLs) in a face-to-face configuration $[12,13]$. Free-running SLs behave as damped oscillators whose internal frequency of intensity pulsations lies in the

\footnotetext{
*Also at Frankfurt Institute for Advanced Studies (FIAS), RuthMoufang-Strasse 1, 60438 Frankfurt am Main, Germany.

†laserdynamics@gmail.com
}

gigahertz range. Consequently, and due the finite speed of light, the interaction between two or more SLs coupled face to face at distances of millimeters or longer must be described in terms of delayed couplings. This is particularly important when the interaction between the systems is bidirectional or in general subject to feedback loops. It is important to note here that delayed couplings have turned out to have relevance in various other systems, e.g., in slime molds [14], and very prominently in neurons in the brain $[15,16]$.

Despite the paradigmatic character of the configuration, delay-coupled oscillators were first studied only at the end of the 1980s [17] and, with respect to lasers, two face-to-face delay-coupled SLs were studied only at the end of the 1990s. Experimental and numerical studies have demonstrated that the coupling-induced dynamics of two identical lasers might spontaneously result in a temporal symmetry breaking [13,18-25]. When two semiconductor lasers are interacting through the mutual injection of their coherent optical fields, instead of showing identical behavior, the two twin lasers develop an achronal generalized synchronization between them. This type of synchronization is characterized by the appearance of significant peaks at $\pm \tau$ in the cross-correlation function between the laser intensities, with $\tau$ being the coupling time $[13,21-23]$. The coupling-induced dynamics of systems that are bidirectionally and symmetrically coupled do not synchronize at zero lag, although the zero-lag identical synchronization is a mathematical solution. However, it turns out not to be stable. This has been particulary analyzed for two bidirectionally coupled SLs [26-28].

One of the first proposals to obtain zero-lag synchronization between the dynamics of three SLs placed far apart was given by Sivaprakasam and co-workers [29]. In their scheme a SL subject to optical feedback is bidirectionally coupled to a free-running laser [30]. When the output of the second laser is unidirectionally coupled to a third one, the time shift can be compensated by the propagation distance, and even 
be nullified. More recently, Yamamoto et al. introduced another configuration, based on a central laser, unidirectionally injecting two outer lasers with equal relaxation oscillation resonances. The two outer lasers exhibited synchronized emission, with higher correlation among them than to the driver [31].

From a different perspective, investigations of three instantaneously coupled semiconductor or solid-state lasers interacting through their overlapping optical fields were performed by Winful [32] and Roy and collaborators [33]. These authors found that, when arranged in a linear chain, an identical synchronization between the first and third lasers appears, while the temporal traces of either of the outer lasers and the central one are rather uncorrelated.

All these various findings call for a deeper study of how the introduction of finite communication time delays between the lasers affects or shapes the synchronization properties of interacting SLs and other oscillators. In particular, it was not obvious whether lasers interacting through the same topology of mutual connections but with a non-negligible delay time were able to synchronize at all.

To address these issues, we study here the synchronization characteristics of three delay-coupled semiconductor lasers arranged in an open-end (linear chain) configuration. In particular, we extensively review the main properties of the zero-lag synchronization between the outer elements of the array that has been reported experimentally and numerically in Ref. [34]. There, it was demonstrated that even in the presence of long coupling delays the dynamics of the first and third laser self-organized to produce an identical and isochronous signal. Surprisingly, the central laser, which mediates in the communication between the outer ones, was found to correlate with the dynamics of the outer lasers, lagging behind by the coupling delay. In addition, we demonstrated that the onset of the zero-lag synchronization is independent of the particular choice of oscillator and can also be demonstrated in a similar configuration of delay-coupled neurons [34]. We identified the requirement for the robust zero-lag solution as having a central relay element which equally redistributes the incoming signals to both outer elements [35]. Since then, various other works have supported this finding, demonstrating zero-lag synchronization in similar coupling configurations with other oscillating elements [36-38] and analyzing the stability of the zero-lag solution $[39,40]$.

After introducing the modeling equations and parameters in Sec. II, we present our main result in Sec. III. We focus on the open-end configuration of three mutually delay-coupled SLs. The characterization of the coupling-induced instabilities, as well as the transition from the two-laser to the threelaser setups is explored there. The leader and laggard roles of the relay element are also analyzed as a function of the coupling strength. Section IV is devoted to assessing the robustness of the synchronization solution by exploring the effects of different mismatches and dynamical perturbations. Finally, a discussion of our results in comparison to other setups studied in the literature is presented in the Discussion section.

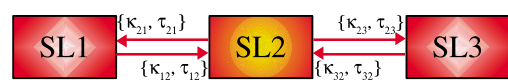

FIG. 1. (Color online) Open-end scheme of three mutually coupled semiconductor lasers interacting bidirectionally with a time delay.

\section{MODEL}

The system under investigation consists of three singlemode SLs coupled in a linear array configuration via the delayed mutual injection of their lasing modes. For numerical purposes the modeling is performed at the level of rate equations according to Ref. [12], which take into account the different delayed coupling terms between the SLs.

The adaptation of such a modeling to the case of three mutually-coupled SLs interacting in an open-end configuration (as sketched in Fig. 1) reads

$$
\begin{gathered}
\dot{E}_{1}=i \Delta \omega_{1} E_{1}+\frac{1}{2}(1+i \alpha) G\left(N_{1},\left\|E_{1}\right\|\right) E_{1}+\kappa_{21} \exp \left(i \phi_{21}\right) E_{2}(t \\
\left.-\tau_{21}\right), \\
\dot{N}_{1}=\frac{I_{1}}{e}-\gamma_{e} N_{1}-G\left(N_{1},\left\|E_{1}\right\|\right)\left\|E_{1}\right\|^{2} \\
\dot{E}_{2}=i \Delta \omega_{2} E_{2}+\frac{1}{2}(1+i \alpha) G\left(N_{2},\left\|E_{2}\right\|\right) E_{2} \\
+\kappa_{12} \exp \left(i \phi_{12}\right) E_{1}\left(t-\tau_{12}\right)+\kappa_{32} \exp \left(i \phi_{32}\right) E_{3}\left(t-\tau_{32}\right), \\
\dot{N}_{2}=\frac{I_{2}}{e}-\gamma_{e} N_{2}-G\left(N_{2},\left\|E_{2}\right\|\right)\left\|E_{2}\right\|^{2}, \\
\dot{E}_{3}=i \Delta \omega_{3} E_{3}+\frac{1}{2}(1+i \alpha) G\left(N_{3},\left\|E_{3}\right\|\right) E_{3} \\
+ \\
+\kappa_{23} \exp \left(i \phi_{23}\right) E_{2}\left(t-\tau_{23}\right), \\
\dot{N}_{3}=\frac{I_{3}}{e}-\gamma_{e} N_{3}-G\left(N_{3},\left\|E_{3}\right\|\right)\left\|E_{3}\right\|^{2},
\end{gathered}
$$

where $E_{m}(t)$ is the complex amplitude of the optical field generated by laser $m$ and $N_{m}$ represents the corresponding carrier number. $\|\cdots\|$ denotes the amplitude of the complex field. The nonlinear gain function $G\left(N_{m},\left\|E_{m}\right\|^{2}\right)$ is given by

$$
G\left(N_{m},\left\|E_{m}\right\|\right) \equiv \frac{g\left(N_{m}-N_{0}\right)}{1+s\left\|E_{m}\right\|^{2}}-\gamma .
$$

Initially, most of the internal laser parameters are assumed identical for the three lasers and are the same as in [34]; the linewidth enhancement factor $\alpha=3$, the differential gain $g$ $=1.2 \times 10^{-5} \mathrm{~ns}^{-1}$, the transparency value for the carrier number $N_{0}=1.25 \times 10^{8}$, the saturation coefficient $s=5 \times 10^{-7}$, the photon decay rate $\gamma=496 \mathrm{~ns}^{-1}$, and the carrier decay rate $\gamma_{e}=0.651 \mathrm{~ns}^{-1}$. With these internal parameters the threshold current of the three lasers is $I_{\mathrm{th}}=17.35 \mathrm{~mA}$. In the former equations, the reference frequency for the slowly varying amplitude of the electric fields is chosen to be $\Omega=\left(\omega_{1}+\omega_{2}\right.$ $\left.+\omega_{3}\right) / 3$, where $\omega_{i}$ is the central frequency of each solitary 
laser. Detunings $\left(\Delta \omega_{i}\right)$ are taken with respect to $\Omega$. Unless other coupling conditions are explicitly mentioned, the coupling strengths and delay times are assumed to be identical in the two coupling branches of Fig. $1 ; \kappa_{12}=\kappa_{21}=\kappa_{32}=\kappa_{23}$ $=20 \mathrm{~ns}^{-1}$ and $\tau_{12}=\tau_{21}=\tau_{32}=\tau_{23}=3.65 \mathrm{~ns}$. The chosen coupling delays correspond to a physical separation between each pair of lasers of more than $1 \mathrm{~m}$. In addition, to limit the parameter space, we restrict the propagation phases to be equal, with $\phi_{12}=\phi_{21}=\phi_{32}=\phi_{23}=0 \mathrm{rad}$. Nevertheless, we have checked that the choice of $\phi_{i j} \neq 0$ and also choosing them as having different values do not change the qualitative features of the dynamical behavior found.

For the numerical integration, a second order RungeKutta method adapted to handle delay-differential equations with multiple constant delays is used in all the simulations presented here [41].

\section{DYNAMICS OF THREE SEMICONDUCTOR LASERS IN AN OPEN-END ARRAY}

\section{A. Coupling-induced instabilities}

In order to explore the instabilities emerging from the mutual coupling of three semiconductor lasers interacting with a time delay, we first set the lasers to operate in a highly symmetric configuration namely, within a perfect freerunning frequency matching $\left(\Delta \omega_{1}=\Delta \omega_{2}=\Delta \omega_{3}=0\right)$ and a moderate and identical current pump $I=I_{1}=I_{2}=I_{3}=27.5 \mathrm{~mA}$. With this level of pumping the solitary relaxation oscillation frequency (ROF) of the three lasers amounts to $4.33 \mathrm{GHz}$.

Once coupled under these conditions, the three semiconductor lasers are observed to enter into a dynamical regime similar to the coherence collapse (CC) $[42,43]$. In this regime the optical intensity of each laser fluctuates in a chaotic manner. Remarkably, after some transient, the chaotic traces of the outer lasers, i.e., SL1 and SL3, although initially uncorrelated, start to become more and more alike up to the point

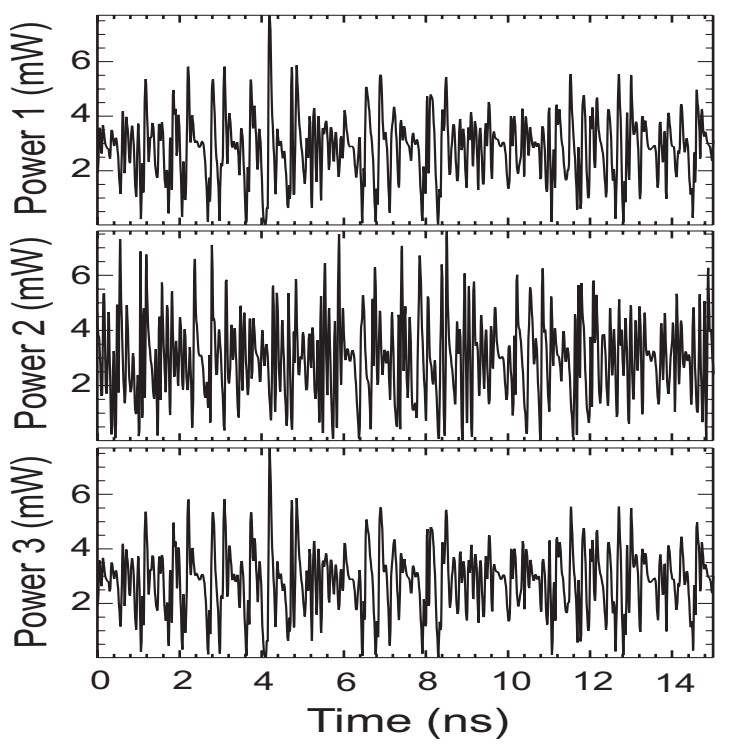

FIG. 2. Temporal traces of the optical intensity of the three lasers in the open-end configuration. $\kappa=20 \mathrm{~ns}^{-1}$ and $\tau=3.65 \mathrm{~ns}$.
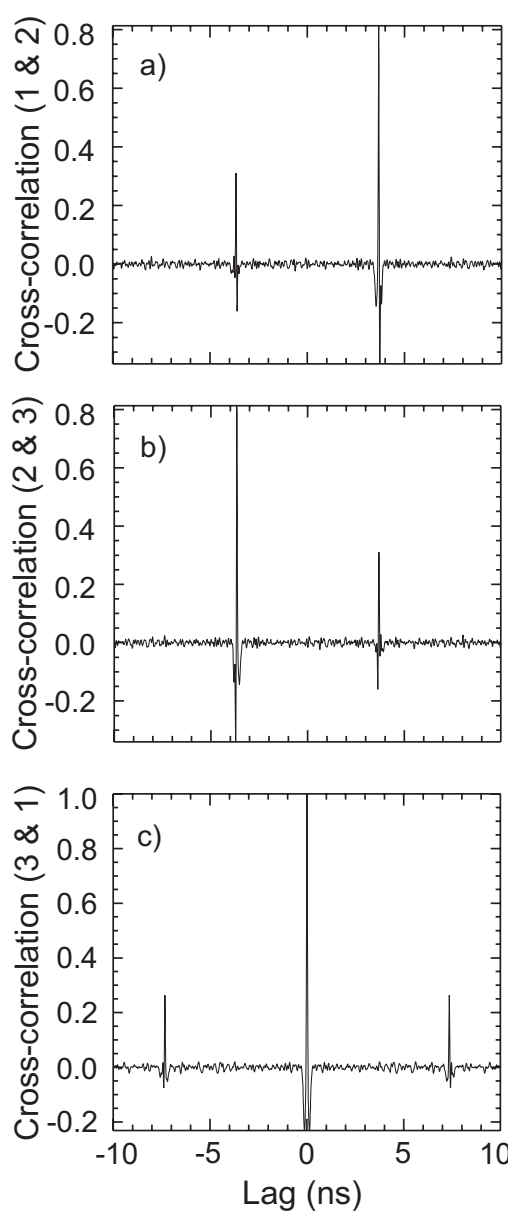

FIG. 3. Cross-correlation functions for the open-end configuration with three lasers. $\kappa=20 \mathrm{~ns}^{-1}$ and $\tau=3.65 \mathrm{~ns}$.

that they end up being perfectly synchronized without any lag [34]. Numerically computed traces of the intensity dynamics of the three lasers demonstrating this effect are shown in Fig. 2. To check the statistical significance of this synchronization phenomena we have simulated Eqs. (1)-(6) starting from various nonidentical initial conditions for the lasers. All the simulations returned the same results: zero-lag synchronization of the lasers occupying the outer positions. At this point it is worth remembering that the symmetry under the exchange between lasers 1 and 3 only assures the existence of a synchronized solution, but not its stability [44]. Remarkably, the stability of the zero-lag solution is maintained even in the presence of arbitrarily long coupling delays [34,39]. Also, considering spontaneous emission noise in the rate equations only slightly reduces the correlations between the outer lasers.

The cross-correlation analysis between the intensity time series additionally reveals a considerable degree of similarity between the traces of either of the outer lasers and the central one. In Fig. 3 it is observed that, once properly shifted, a significant degree of correlation $(\sim 0.8)$ exists between the traces of either of the lasers occupying the outer positions and the laser 2. The lag for which the maximum of the crosscorrelation function appears corresponds to the coupling time $\tau$. The asymmetric cross-correlation function between SL1 (or SL3) and SL2 indicates that the outer lasers are advanc- 

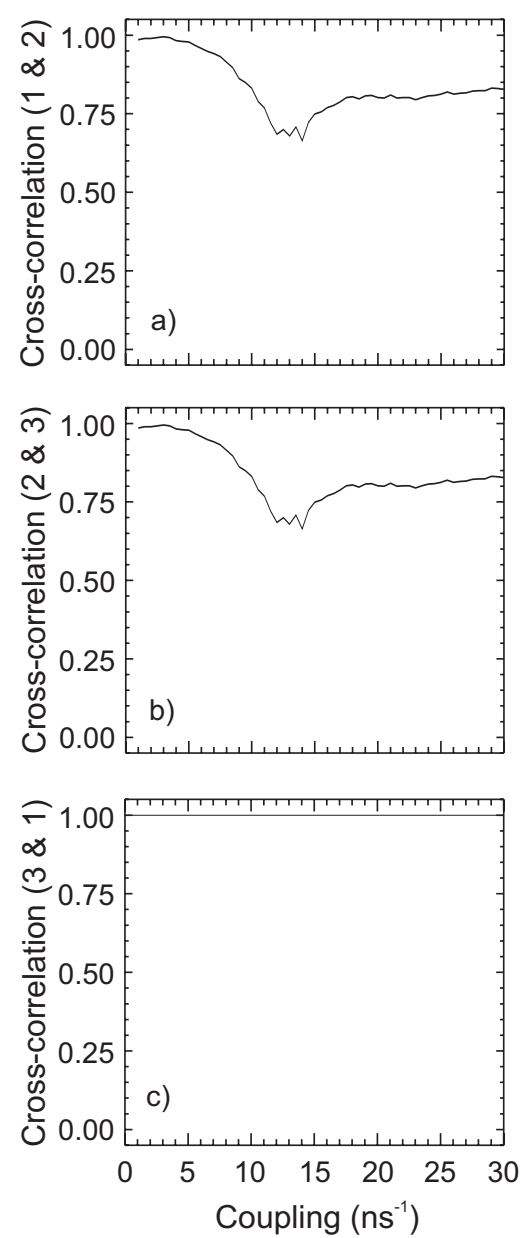

FIG. 4. Maximum of the cross-correlation function between pairs of SLs as a function of their common coupling strength $\kappa$.

ing the dynamics of the central one by the coupling delay $\tau$. Thus, remarkably, the mediator element is lagging behind the synchronized outer units for which it is acting as a relay. This type of dynamics excludes the interpretation of the central element as a simple master slaving the outer lasers, and illustrates the collective self-organized dynamics of the network of lasers induced by the mutually reciprocal connections.

\section{Influence of coupling strength}

In the following we investigate how this synchronization scenario depends on the coupling strength between lasers. We proceed by adiabatically increasing the coupling strength between the three SLs and computing the dynamics for each value of the coupling rate. The common coupling strength $\kappa=\kappa_{12}=\kappa_{21}=\kappa_{23}=\kappa_{32}$ is varied from 0 to $30 \mathrm{~ns}^{-1}$. The common coupling delay time is maintained constant at $\tau$ $=3.65 \mathrm{~ns}$. For each time series, we determine the maximum of the cross-correlation function between pairs of lasers. The results in dependence on the coupling strengths are shown in Fig. 4. It is worth mentioning that for most of the coupling strength values the three lasers operate in the hyperchaotic $\mathrm{CC}$ regime although some windows of periodic behavior are also observed for small coupling rates. Interestingly, regard- less of the dynamics exhibited by the lasers, the perfect synchronization at zero lag between the outer units extends to the whole range of couplings explored. No coupling threshold for the transition from unsynchronized to synchronous motion is observed for this pair of lasers which achieve the synchronization state for arbitrarily small couplings (a different scenario can appear when the outer lasers are simultaneously subject to feedback from an external reflector and consequently develop their own dynamics even when uncoupled). The central laser, on the other hand, exhibits changes of the correlation coefficient with the outer units from a value close to 1 obtained for small couplings and periodic dynamics to a value around $\sim 0.8$, which is attained for large coupling strengths, a regime in which chaotic dynamics develops. We also found that from coupling rates $\kappa$ $\geq 6 \mathrm{~ns}^{-1}$ the lag between SL2 and SL1 (or SL3) stabilizes around $\tau$, i.e., the central laser lags behind the outer ones. For small coupling strengths, where the dynamics is periodic, the cross-correlation function turns out to be rather symmetric and a leader-laggard behavior cannot be clearly distinguished.

\section{Transition from the two-laser case to the three laser-chain}

The different synchronization properties of setups with different numbers of lasers (two and three coupled lasers have been mainly considered in the literature) motivated us to study how such a transition occurs. To better understand the link from the two-coupled laser problem to our case with three interacting lasers, we performed the following numerical simulation. Instead of symmetrically increasing the coupling strength between the three lasers we start from a configuration where only SL1 and SL2 are interacting with a constant coupling strength $\left(\kappa_{12}=\kappa_{21}=20 \mathrm{~ns}^{-1}\right)$. Laser 3 is put into play by continuously increasing the coupling rates $\kappa_{23}$ $=\kappa_{32}$ from 0 to $30 \mathrm{~ns}^{-1}$. Therefore, we can study in detail the transition from the known cases of leader-laggard dynamics for two lasers to the zero-lag synchronization situation for three lasers. In Fig. 5 the correlation properties of the solutions are shown.

We observe that the maximum of the cross-correlation function between the outer lasers increases with the coupling strength up to the value of $\kappa_{23}=\kappa_{32}=20 \mathrm{~ns}^{-1}$, i.e., the degree of correlation is maximum in the most symmetrical situation. The lag between SL1 and SL3, determined via the maximum of the cross-correlation function, is again zero for most of the coupling rates investigated. Regarding the role of the central laser, one can see that the amount of correlation between either of the outer lasers and the central one also grows, exhibiting a peak at $\kappa_{23}=\kappa_{32}=20 \mathrm{~ns}^{-1}$. After that point, the correlation between SL1 and SL2 starts to decrease, while the correlation between lasers 2 and 3 saturates at a value around $\sim 0.7$. It is interesting to note that, for example, at $\kappa_{23}=\kappa_{32}=30 \mathrm{~ns}^{-1}$, the interaction between lasers 2 and 3 is stronger than when the coupling is set at $\kappa_{23}=\kappa_{32}=20 \mathrm{~ns}^{-1}$ and nevertheless, the correlation between these two lasers is higher in this last case for symmetry reasons. Regarding the lag analysis, we observe that for moderate to large coupling values $\left(\gtrsim 10 \mathrm{~ns}^{-1}\right)$ both outer lasers advance the dynamics of the central one by a time $\tau$. 

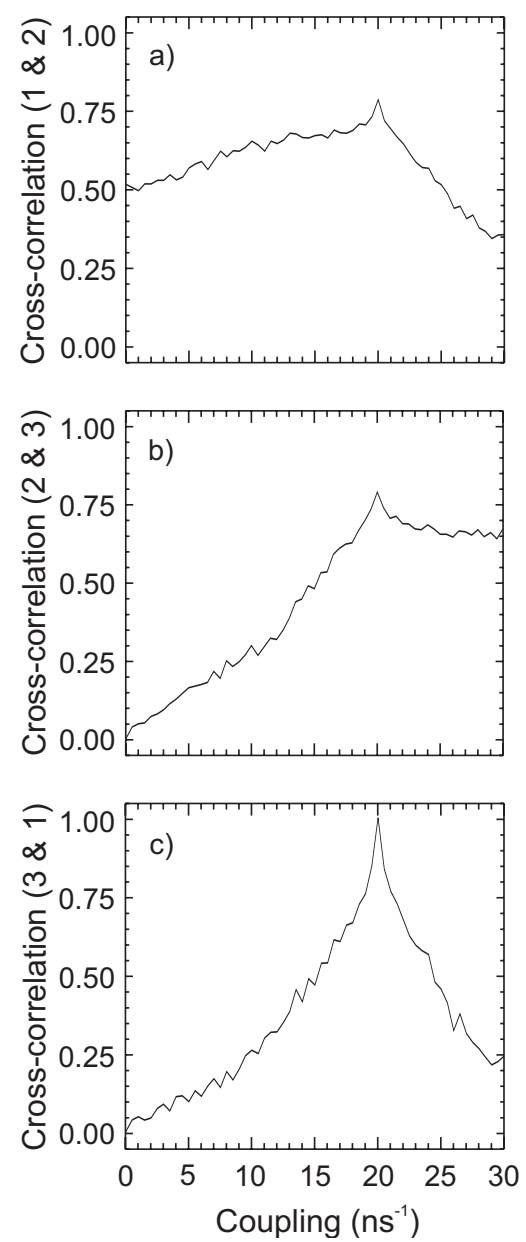

FIG. 5. Maximum of the cross correlation between the pairs of SLs as a function of the coupling strength between SL2 and SL3 $\left(\kappa_{23}=\kappa_{32}\right)$.

So far we have considered only symmetric mutual interactions between any pair of lasers. This is, for any pair of lasers $(n, m)$ the coupling strength was such that $\kappa_{n m}=\kappa_{m n}$. In such situations the central laser is always receiving more optical injection than the outer ones because it is the only one that is simultaneously linked to two lasers. To check whether the synchronization solutions we have observed until now are maintained when this situation is modified, e.g., when SL2 receives less optical injection than SL1 and SL3, we perform the following numerical simulations. We fix the coupling strengths from SL2 to the outer lasers at $\kappa_{21}=\kappa_{23}$ $=20 \mathrm{~ns}^{-1}$, and scan the input coupling of SL2 from $\kappa_{12}$ $=\kappa_{32}=0$ up to $30 \mathrm{~ns}^{-1}$. The correlation analysis is presented in Fig. 6.

We find that the synchronization between SL1 and SL3 at zero lag is stable under all considered conditions. Regarding the leader-laggard role between any of the outer lasers and the central one, we find a change of role occurring at $\kappa_{12}$ $=\kappa_{32} \sim 10 \mathrm{~ns}^{-1}$. This is the symmetric situation in which the center laser gets in total as much input as the outer lasers. We first analyze the case for coupling strengths below such a critical rate. In that region the cross-correlation function between any of the outer lasers and SL2 typically shows two peaks at $\pm \tau$. However, in such functions the peak associated
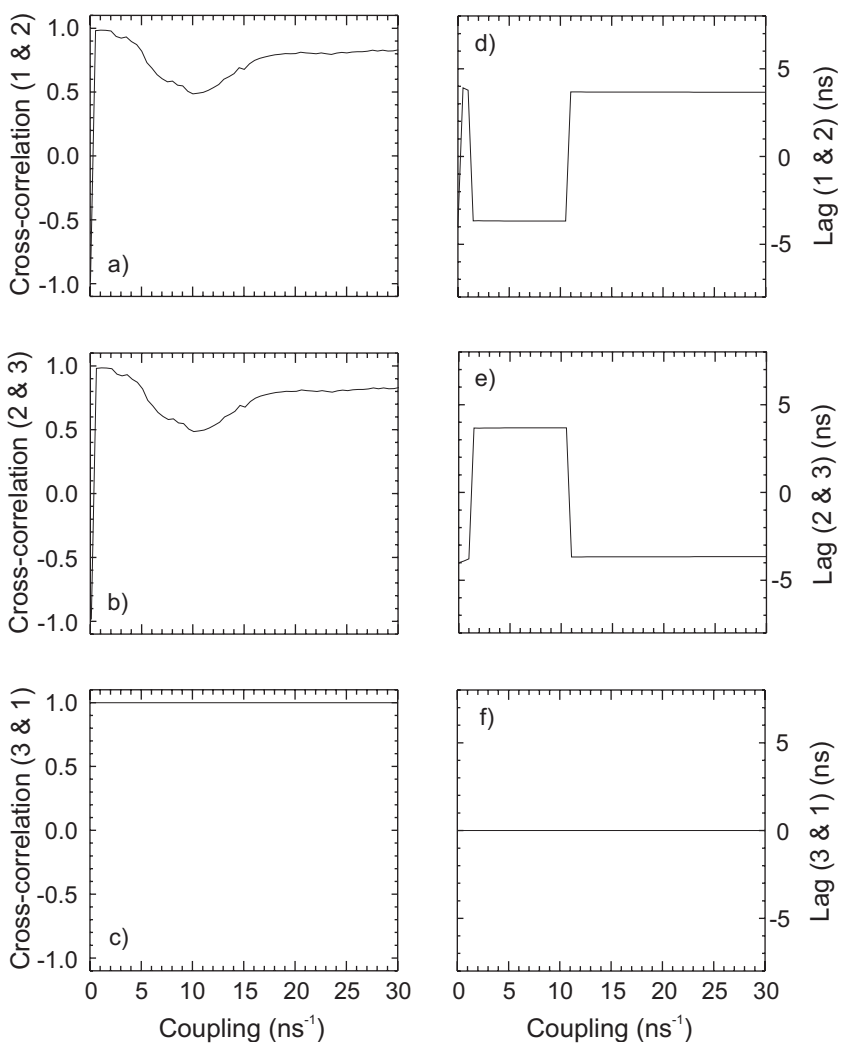

FIG. 6. Maximum of the cross correlation between the pairs of SLs and lag at which this maximum appears as a function of the central laser input strength $\left(\kappa_{12}=\kappa_{32}\right)$.

with the situation in which the central laser advances the extreme ones is only a bit higher than the other peak, which is related to the situation that the outer lasers are advancing the central one. Since the difference between the two peaks is comparatively small it becomes difficult to speak about a true leader-laggard dynamics. For coupling strengths above $\sim 10 \mathrm{~ns}^{-1}$, the difference between peaks is much larger than in the previous case, hence indicating a well-posed leaderlaggard dynamics in which the central laser lags. For this large-coupling regime, the dynamics of SL1 and SL3 are always found to advance the central one by the injection delay time $\tau$.

It is worth elaborating at this point more on the situation $\kappa_{12}=\kappa_{32}=10 \mathrm{~ns}^{-1}$ and $\kappa_{21}=\kappa_{23}=20 \mathrm{~ns}^{-1}$ in which the center laser gets in total as much input as each of the outer lasers. Since we do find zero-lag synchronization of SL1 and SL3, the dynamics is in this case identical to the case of two mutually delay-coupled lasers, as discussed in [13]. SL2 receives as much delayed coupling as SL1 and SL3 separately. Our results reported here are indeed fully compatible with the earlier interpretation of generalized synchronization between neighbored delay-coupled lasers, the instability of the zero-lag synchronization of SL2 with respect to SL1 and SL3 and the determination of the leader-laggard role via detuning. Under these conditions zero detuning represents the switching point for the center laser to lead or lag in its dynamics as in [13]. For the situation, as studied mostly in this manuscript, that $\kappa_{12}=\kappa_{12}=\kappa_{23}=\kappa_{32}$ large detunings are required in order to switch the leader-laggard role between center and 

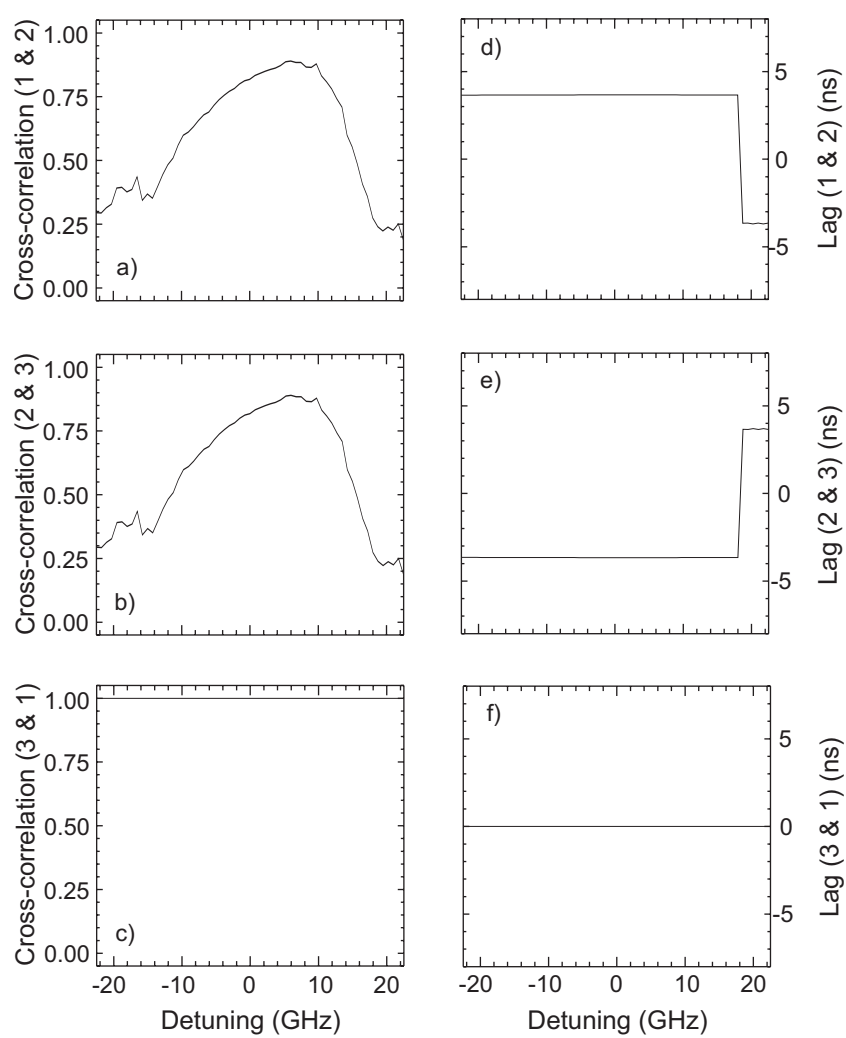

FIG. 7. Maximum of the cross correlation between the pairs of SLs and lag at which this maximum appears as a function of the central laser frequency deviation, $\Delta \omega_{2}$.

outer lasers. The influence of detuning is discussed in more detail in the following section.

\section{B. Robustness of the synchronized solutions}

\section{Influence of the detuning}

The ability of a set of oscillators to synchronize is strongly dependent on how far their solitary frequencies are. Considerations of detuning leads to the concept of Arnold tongue. For laser oscillators the role of a moderate detuning between the free-running optical frequencies of two coherently coupled edge-emitting lasers has been found to establish a leader-laggard role, with the high-frequency laser advancing the dynamics of its counterpart [13,21]. Since a small detuning is almost unavoidable in real experiments, there is a strong motivation to observe the effects of detuning in an open-end array of lasers.

We recover here the first considered (standard) coupling conditions by setting all the coupling constants at $\kappa=\kappa_{12}$ $=\kappa_{21}=\kappa_{23}=\kappa_{32}=20 \mathrm{~ns}^{-1}$, and the delay times at $\tau=\tau_{12}=\tau_{21}$ $=\tau_{23}=\tau_{32}=3.65 \mathrm{~ns}$. We start with the case where the outer lasers share a common solitary frequency $\left(\Delta \omega_{1}=\Delta \omega_{3}=0\right)$ and we only allow for a detuning with respect to the central laser $\left(\Delta \omega_{2} \neq 0\right)$. The central laser frequency detuning is scanned from -22.5 up to $22.5 \mathrm{GHz}$ in Fig. 7. The graphics show that the zero-lag synchronization between SL1 and SL3 is perfectly maintained in all the range of values considered for the detuning. The data reported in Ref. [34] sup- port this view since experimentally the zero-lag synchronization was observed up to several tens of gigahertz. In most cases, both outer lasers advance the dynamics of the central one and only for very high positive detunings this lag changes its sign. However, looking in more detail at this hypothetical reversal of the leader-laggard role we must notice that it occurs for a value of the detuning where the linear correlation between the central and outer lasers is almost lost (at $\Delta \omega_{2}=18 \mathrm{GHz}$ the maximum of cross-correlation function is just $\sim 0.2$ ). In fact, around $\pm \tau$ the cross-correlation function also presents two negative peaks reaching the value of $\sim-0.5$ (not seen in Fig. 7 since there only the absolute maxima of the cross-correlation functions are represented), hence indicating that actually an anticorrelated dynamics with time lag between the central and the outer lasers is taking place. Nevertheless, it is important to emphasize here again that for the condition in which the input and output couplings of the central laser are equal $\left(\kappa_{12}+\kappa_{32}=\kappa_{23}=\kappa_{21}\right)$ the switching of roles between leader and laggard occurs at zero detuning.

If a detuning occurs between the outer units $\left(\Delta \omega_{1}-\Delta \omega_{3}\right.$ $\neq 0$ ), we have numerically tested that the synchronized solution is much more sensitive. The robustness of the isochronal sync between SL1 and SL3 is observed to amount to a few hundreds of $\mathrm{MHz}$ (see Fig. 8). The relative dynamics, i.e., the leader-laggard role if any, between the three lasers outside the zero-detuning point becomes difficult to interpret since the correlation coefficient drops very fast down to zero.

\section{Influence of pumping mismatch}

Another important control parameter in this system is the electrical pump current. The current injected into a semiconductor laser can be very well controlled and it is important to check the stability of the synchronization solution upon current mismatches between the coupled lasers. Different dynamical regimes and synchronization characteristics only appear at certain values of the injection current (low-frequency fluctuations, coherence collapse, regular pulse packages, etc.). For these reasons in this section we assess the effect of different pumping levels for each laser and evaluate the robustness of the synchronization solution. As in the previous study of the detuning effect, the coupling strengths are fixed at $\kappa_{12}=\kappa_{21}=\kappa_{23}=\kappa_{32}=20 \mathrm{~ns}^{-1}$, and the delay times at $\tau_{12}$ $=\tau_{21}=\tau_{23}=\tau_{32}=3.65 \mathrm{~ns}$.

We first proceed by varying the bias current of the central laser $I_{2}$ from 17.5 to $37.5 \mathrm{~mA}$ while keeping the outer laser pumps constant at $I_{1}=I_{3}=27.5 \mathrm{~mA}$. We find that the only effect of increasing $I_{2}$ is to monotonically decrease the correlation level between respective neighbors. However, the degradation of the synchronization quality captured by the correlation coefficient is not severe and it decreases from a value of almost 1 for periodic regimes down to $\sim 0.7$ for chaotic states. It is worth noting that the maximum of the correlation between SL2 and SL1 (or SL3), is not attained in the symmetric configuration where all three lasers are operating at $27.5 \mathrm{~mA}$ but at pump levels of the central laser close to the threshold. These results together with Fig. 4 are indicative of the very low optical power that is needed to bring the outer elements SL1 and SL3 into synchrony. Regarding the 

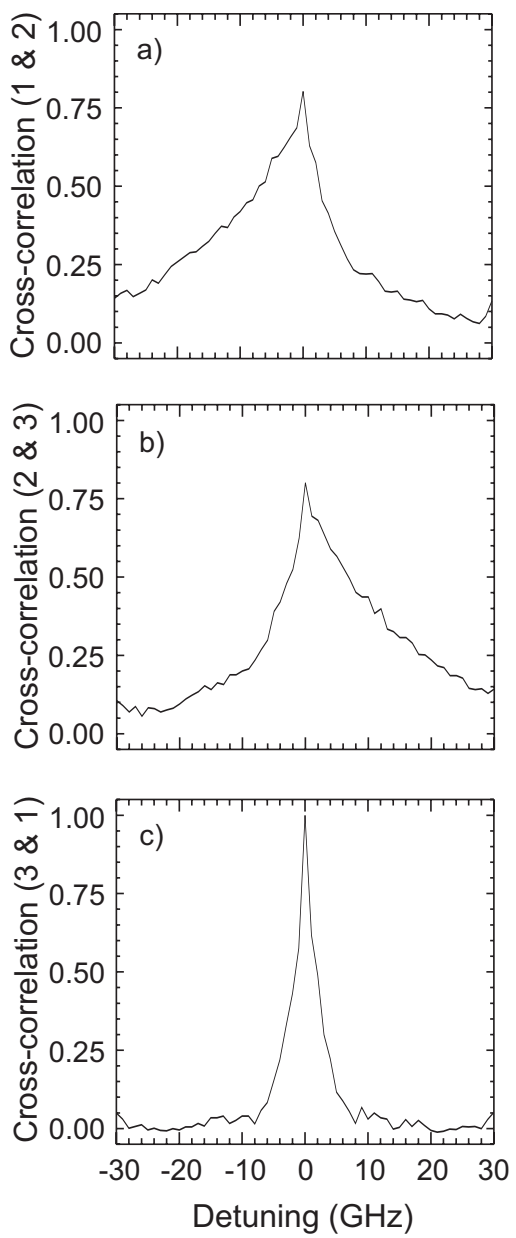

FIG. 8. Maximum of the cross-correlation function between the pairs of SLs as a function of the detuning between the outer lasers, $\left(\Delta \omega_{1}-\Delta \omega_{3}\right)$.

timing of the synchronized solutions, we find that the lags at which the maxima of the cross-correlation functions appear do not shift with the pump level.

Next, we consider pump mismatches between the lasers occupying the outer positions, i.e., SL1 and SL3. The effects on the synchronization are much more severe now. The results are summarized in Fig. 9 where the bias current of SL3 is scanned from 17.5 to $37.5 \mathrm{~mA}$. There the levels of SL1 and SL2 are fixed to $27.5 \mathrm{~mA}$. In such a case high correlation between the outer lasers is maintained only within a range of a few miliamperes wide. For small pump deviations, series of bursts of desynchronization perturb the identical synchronization solution between SL1 and SL3. Nevertheless, the location of the maximum of the cross correlation function between SL1 and SL3 remains at zero lag.

\section{Robustness under dynamical perturbations}

In the preceding sections we have demonstrated that the synchronization between the extreme lasers is only moderately robust against a mismatch between them. In contrast, the effect of mismatches between the central laser and the outer units turned out to be hardly significant and the synchronization between SL1 and SL3 was found to be ex-
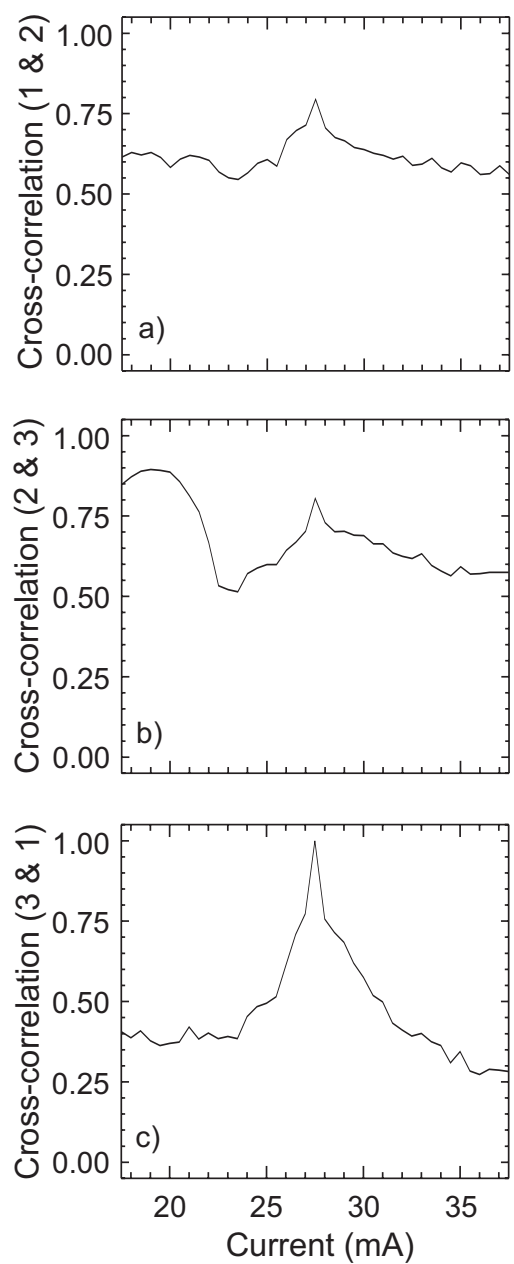

FIG. 9. Maximum of the cross correlation between the pairs of SLs as a function of the current level of laser 3.

tremely robust upon changes in the parameters of central laser [35].

These results have been obtained under the conditions of stationary mismatches, i.e., once the differences in the natural frequencies or pumping levels were applied they were kept constant throughout the temporal evolution of the lasers. In this section, we investigate the effects of a dynamical perturbation on the synchronization properties. To do so, we simulate a pulse of current in one of the lasers and study how this perturbation propagates in the system and affects the synchronization solution and how fast synchronization can be recovered after the perturbation.

Within the standard and symmetrical coupling conditions, we start by generating a current pulse of Gaussian profile with an amplitude of $10 \mathrm{~mA}$ and full width at half maximum of $1.2 \mathrm{~ns}$ in the central laser. This pulse is superimposed to the constant bias drive at $27.5 \mathrm{~mA}$. The time series of the three lasers, the pulse profile, and the synchronization error and sync plot for SL1 and SL3 are depicted in Fig. 10. The synchronization solution between the outer lasers is perfectly maintained, even during the time the perturbation reaches the lasers SL1 and SL3, one coupling time after the pulse was generated. The perturbation remains completely unnoticed from the synchronization point of view. This can be under- 

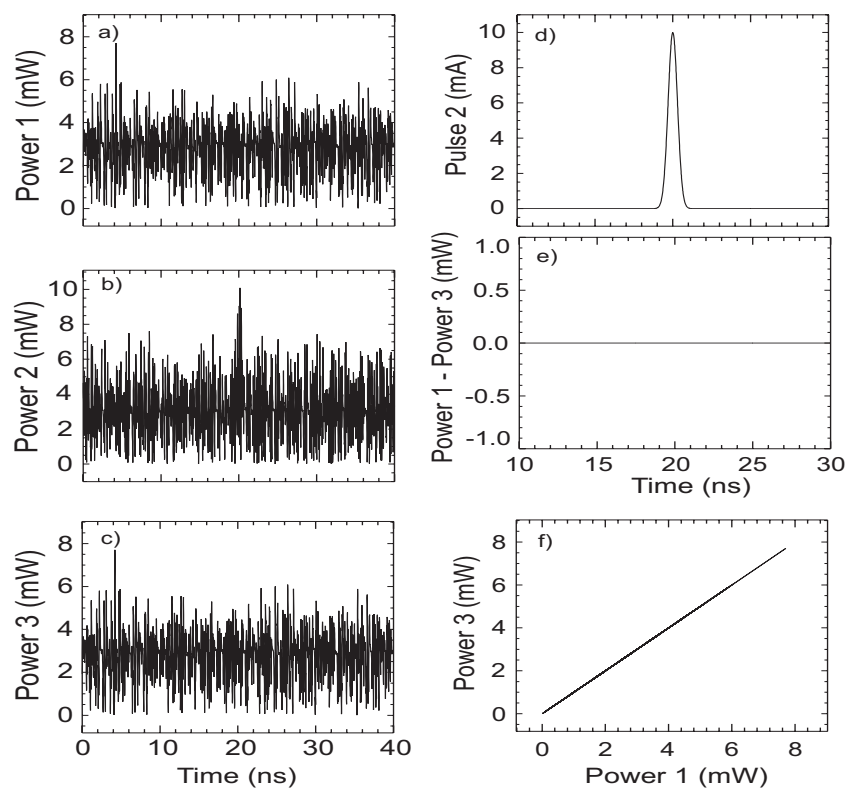

FIG. 10. (a), (b), and (c) contain the temporal traces of the optical power of the three lasers, (d) represents the applied current pulse wave from, while the sync error and sync plot between lasers 1 and 3 are shown in (e) and (f), respectively. Perturbation is applied on SL2.

stood in the sense that such a symmetric perturbation applied to the center laser affects and modifies the synchronization manifold, but does not represent a perturbation transverse to the synchronization manifold.

Perturbations transverse to the synchronization manifold are realized by asymmetrically perturbing one of the outer lasers. We investigated this by applying the same pulse now to one of the lasers occupying the outer positions. The result is depicted in Fig. 11, clearly showing that the synchronization error between lasers 1 and 3 becomes different from zero, but almost only during the pulse duration. This means that just after the perturbation on SL1 ends this laser is almost immediately resynchronizing due to the continuous coupling that it receives from the central laser. There is no need to wait until the pulse is reaching the third laser $(\sim 2 \tau)$ to reinforce synchrony [35]. This result is quite different if compared to the expected resynchronization times for two face-to-face coupled lasers. In such a case, replicas of the original disturbance in the synchronization error are expected for time $\tau$ and multiples of this value after the onset of the pulse generation. In our case, however, once the perturbation reaches the central laser, it is symmetrically distributed toward SL1 and SL3 due to the similar values of the coupling coefficients, again only affecting the synchronization manifold and not the directions transverse to it. This leads to extremely fast synchronization times which in our case amount to the decay time of the ROF, which is usually shorter than any of the propagation delays involved.

In cases where the amplitude of the pulses was increased up to $50 \mathrm{~mA}$ (note that this value is almost double the bias current level) or the width of the pulse enlarged up to $12 \mathrm{~ns}$ (a value much larger than the coupling delay time), the same characteristics were found. Hence, the synchronization be-
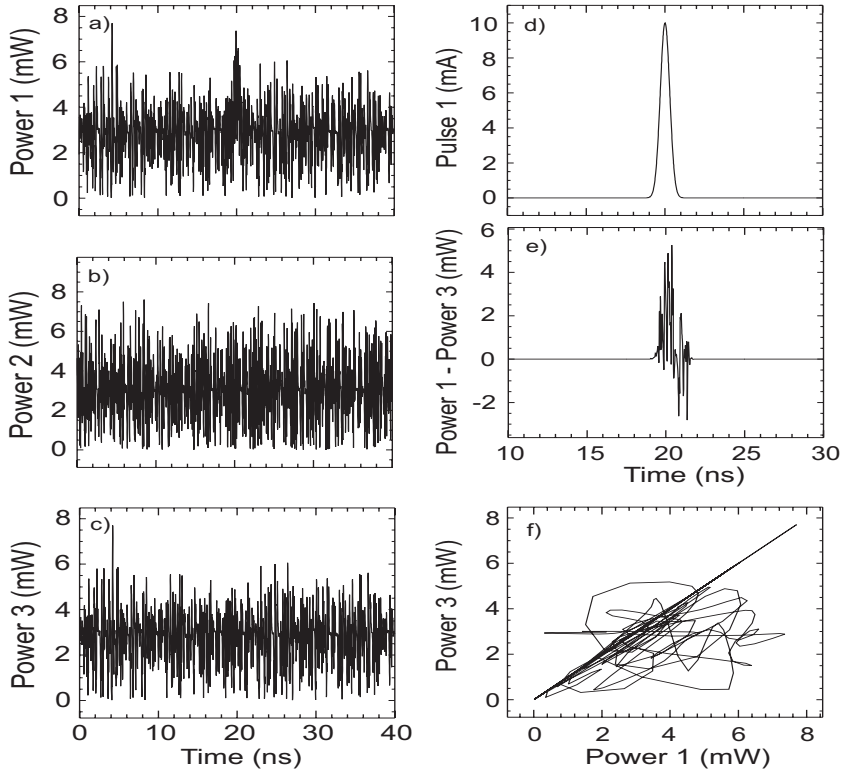

FIG. 11. (a), (b), and (c) contain the temporal traces of the optical power of the three lasers, (d) represents the applied current pulse wave from, while the sync error and sync plot between lasers 1 and 3 are shown in (e) and (f), respectively. Perturbation is applied on SL1.

tween lasers is proven to be quite stable under dynamical perturbations pushing away the solution from the synchronization manifold.

\section{Filtering properties}

If in addition to the collective dynamics of coupled systems an external perturbation is superimposed, the elements of the system can respond in different ways. The characterization of such responses to different types of perturbations is an important property. Among examples of such behaviors one can find regimes where a system can faithfully follow the evolution of an external perturbation and others where the elements filter out the external influence. This reaction is in general dependent on the systems being externally perturbed, as well as the amplitude and frequency content of the perturbation [13]. Important applications in the field of optical cryptography rely on the filtering properties of chaotic lasers [45-47]. For instance, previous studies have exploited selective filtering properties by a SL to decode a message embedded into a chaotic carrier [47-49].

In this section, we study the filtering properties of a chain of three mutually coupled SLs. More precisely, we characterize the degree to which the different systems react to amplify or suppress a given external fluctuation. To this end, we proceed by adding to one of the lasers a sinusoidal modulation with a given frequency $\left[I_{j}=I_{j, \text { bias }}+I_{j, \bmod } \sin \left(2 \pi \nu_{j} t\right)\right]$ to the bias current. The constant component of the currents of all three lasers is set to $27.5 \mathrm{~mA}$, while the coupling constants $\left(\kappa_{12}=\kappa_{21}=\kappa_{23}=\kappa_{32}=20 \mathrm{~ns}^{-1}\right)$ and the delay times $\left(\tau_{12}=\tau_{21}=\tau_{23}=\tau_{32}=3.65 \mathrm{~ns}\right)$ are fixed at typical values. Figure 12 shows the time series of the three lasers and their power spectra, when laser 2 is modulated with an amplitude of $2 \mathrm{~mA}$ at $1.25 \mathrm{GHz}$. 

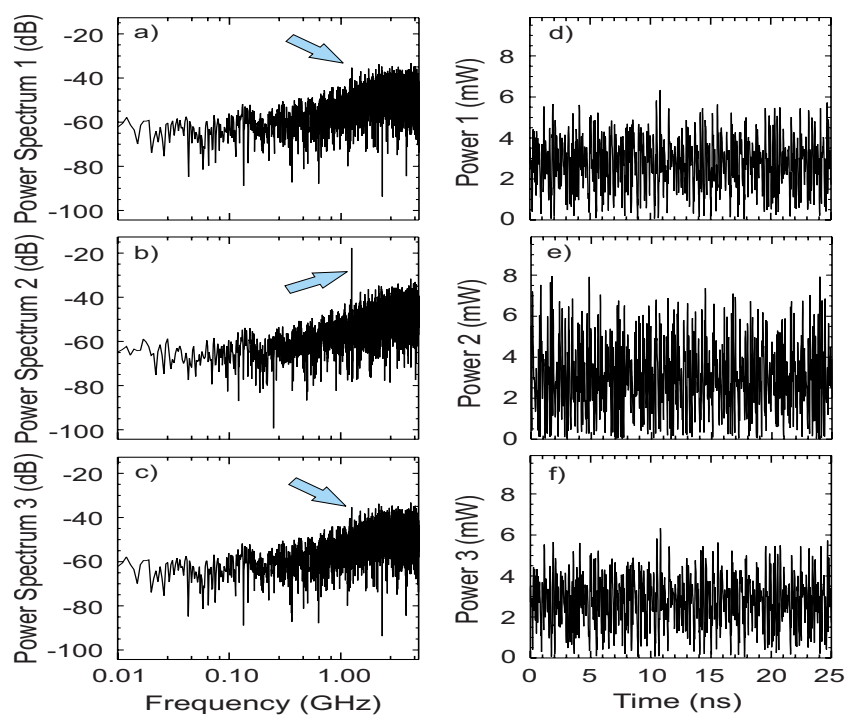

FIG. 12. (Color online) Power spectra and time series of the three lasers. The central laser is modulated at $1.25 \mathrm{GHz}$ with an amplitude of $2 \mathrm{~mA}$. The bias of all three lasers is $27.5 \mathrm{~mA}$. The bias of all three lasers is $27.5 \mathrm{~mA}$. The arrows indicate the peaks related to the modulation of SL2.

A clear peak appears in the spectrum of laser 2 at the frequency of modulation. Smaller peaks also appear at the spectra of lasers 1 and 3 at the same frequency bin. Next, we record the difference in the height of these peaks as a function of the modulation frequency.

We start by scanning the central laser modulation frequency from 0.25 to $5 \mathrm{GHz}$ in $0.25 \mathrm{GHz}$ steps. The pump level has been chosen as $27.5 \mathrm{~mA}$, resulting in a relaxation oscillation frequency of $4.33 \mathrm{GHz}$. We determine the difference in the height of the spectral peaks between the outer

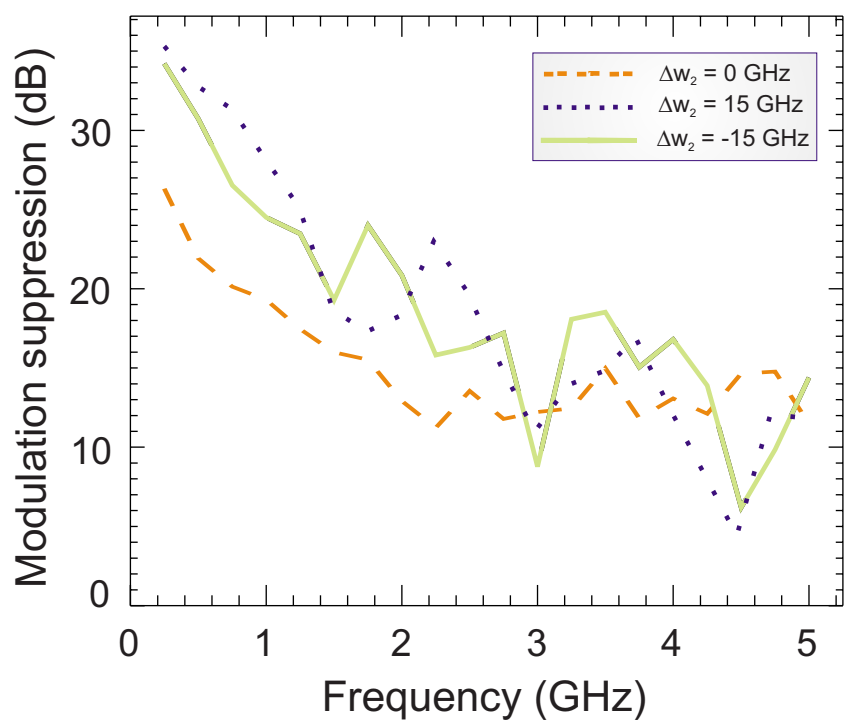

FIG. 13. (Color online) Modulation suppression in dB as a function of the modulation frequency of the central leser. The modulation ratios obtained in lasers 1 and 3 are identical do we represent only one of them. Dashed line, $\Delta \omega_{2}=0 \mathrm{GHz}$; dotted line, $\Delta \omega_{2}$ $=15 \mathrm{GHz}$; solid line, $\Delta \omega_{2}=-15 \mathrm{GHz}$.

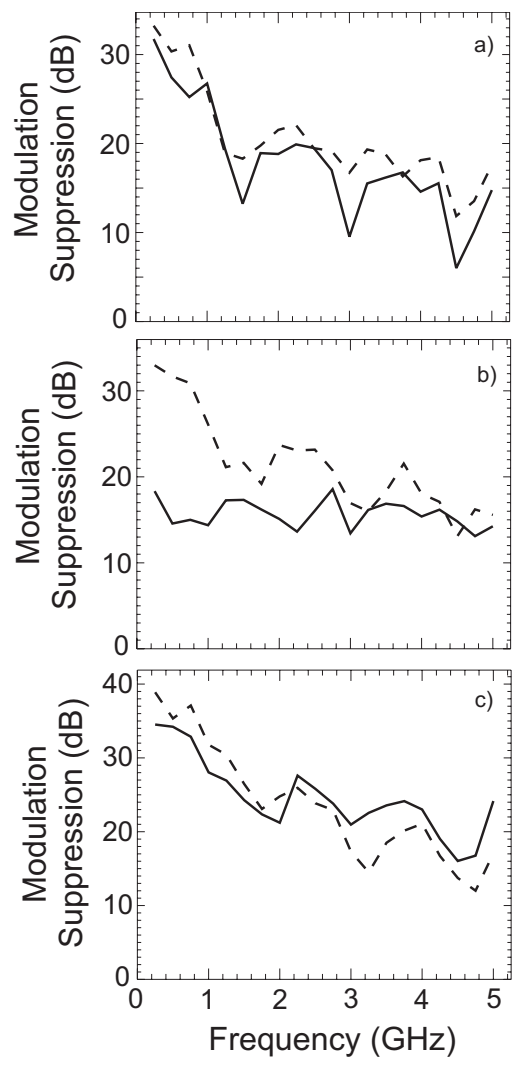

FIG. 14. Modulation suppression in $\mathrm{dB}$ as a function of the modulation frequency of laser 1 . The suppression level at laser 2 (3) is shown as a solid (dashed) line. Top panel, $\Delta \omega_{1}-\Delta \omega_{3}=0 \mathrm{GHz}$, middle panel, $\Delta \omega_{1}-\Delta \omega_{3}=15 \mathrm{GHz}$; bottom, $\Delta \omega_{1}-\Delta \omega_{3}=-15 \mathrm{GHz}$.

lasers and the central one. The results are indicated by the dashed line in Fig. 13. We observe that for the explored range of frequencies a stronger suppression of the perturbation occurs at low frequencies where the suppression rates are quite high. The coefficient of modulation suppression decreases as the frequency increases. Still, for the frequencies explored (up to $5 \mathrm{GHz}$ ) the suppression level stays larger than $10 \mathrm{~dB}$.

In case we allow for a detuning of the free-running optical frequencies between lasers $\left(\Delta \omega_{2} \neq 0\right)$ we can obtain different filtering properties. Figure 13 (dotted line) shows the modulation suppression as a function of the modulation frequency when $\Delta \omega_{2}=15 \mathrm{GHz}$. We find even higher suppression in the low-frequency domain. For the negative detuning case $\Delta \omega_{2}$ $=-15 \mathrm{GHz}$ the modulation suppression coefficient qualitatively shows the same features as its positive counterpart (see solid line of Fig. 13).

Now, we perform the same type of analysis but when laser 1 is modulated (see Fig. 14). For positive detuning, we observe how the ability of the central laser to filter the sinusoidal component coming from laser 1 is maintained at an almost constant level around $16 \mathrm{~dB}$. For negative detuning, however, the suppression ratio of the central laser is significantly higher for lower frequencies and it decays as the modulation frequency increases. The differing suppression behavior depending on the detuning is similar to the behavior described in [13]. A laser lagging in dynamics filters a per- 
turbation more strongly than a laser leading in its dynamics. Another point worth mentioning is that from the lower panel of Fig. 14 we observe that for frequencies higher than $2 \mathrm{GHz}$, the central laser has a better suppression ratio than laser 3. This means that the modulation induced in laser 1 is better reproduced in the distant laser 3 than in its immediate neighbor SL2.

\section{Synchronization for asymmetric coupling times}

So far we have taken both branches of the network module in Fig. 1 to be identical and have chosen the same coupling times. But, will synchronization remain and be stable if different lengths for the paths between lasers are considered?

This question has been investigated by setting the coupling delay times in both branches to be very different. We choose $\tau_{a}=\tau_{12}=\tau_{21}=12 \mathrm{~ns}$ and $\tau_{b}=\tau_{23}=\tau_{32}=3 \mathrm{~ns}$. For moderate coupling strengths, now the synchronization between SL1 and SL3 appears when comparing $P_{1}(t)$ with $P_{3}(t$ $+\Delta \tau)$, where $\Delta \tau=\tau_{b}-\tau_{a}$. This is, the outer laser of the shortest branch (in this case SL3) is able to advance the behavior of the other one (SL1), which is more than 15 ns apart, by the difference of coupling times. Other sets of values for the coupling times have been tested ( $\mathrm{such}$ as $\tau_{a}=\tau_{12}=\tau_{21}=5 \mathrm{~ns}$ and $\tau_{b}=\tau_{23}=\tau_{32}=0.2 \mathrm{~ns}$ ) and in all cases a synchronization solution with lag $\tau_{b}-\tau_{a}$ have been attained between SL1 and SL3. Note that in the latter case, without the presence of a third laser the pairs of mutually-coupled lasers $(1,2)$ and $(2,3)$ would exhibit very different dynamical regimes due to the disparity in the coupling delays between the lasers. Nevertheless, it is a remarkable fact that when all three lasers are present SL1 and SL3 are able to perfectly synchronize [39].

The cross-correlation functions show a multipeak structure. The location of the relative maxima can be related to the delay times and their differences. Figure 15 contains the cross-correlation functions for the three pairs of lasers $(1,2)$, $(2,3)$, and $(3,1)$ when $\tau_{a}=1.5 \mathrm{~ns}$ and $\tau_{b}=3.6 \mathrm{~ns}$. Different delay values have been simulated and in all cases the main peaks of the cross-correlation functions appeared at the lags: $\left(\tau_{a},-\tau_{a}, 2 \tau_{b}-\tau_{a}\right)$ for the pair of lasers $(1,2),\left(\tau_{b},-\tau_{b}, \tau_{b}-2 \tau_{a}\right)$ for the lasers $(2,3)$, and $\left(\tau_{b}-\tau_{a}\right)$ for the pair composed of the outer lasers 3 and 1 .

\section{DISCUSSION AND CONCLUSION}

In this paper we have focused on the problem of three delay-coupled SLs, and more specifically on the properties of the zero-lag synchronization solution found in Ref. [34]. The influence of the coupling strength between the lasers as well as the transition from the two-laser to the three-laser problem has been investigated. At the same time the robustness of the solution has been proven against mismatches in pump level and natural detuning. To test the dynamical robustness of the

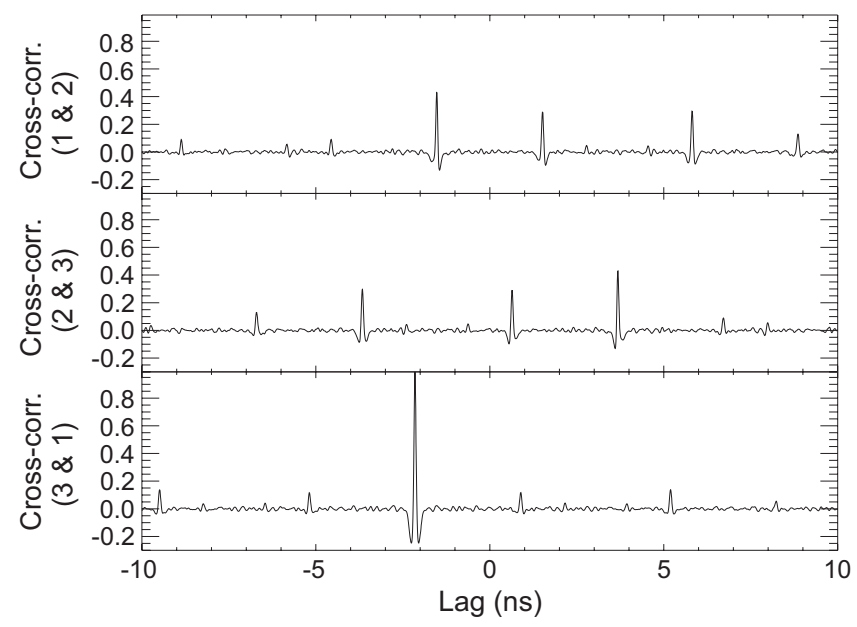

FIG. 15. Cross-correlation function between pairs of lasers with different coupling times. $\tau_{a}=1.5 \mathrm{~ns}$ and $\tau_{b}=3.6 \mathrm{~ns}$. The coupling strength is fixed to $k=20 \mathrm{~ns}^{-1}$.

zero-lag solution we have also applied different types of perturbations. We obtain a strong tendency to fast resynchronization. The filtering properties to a sinusoidal modulation of the current pump of the different lasers and the finding of identical synchronization with time shift in the case of asymmetric coupling delays completed our study.

A different situation was proposed recently by some of us in Ref. [34]. In that paper we reported the observation of zero-lag identical synchronization between two arbitrary distant SLs bidirectionally coupled via a relay element, which can be either another SL or even a passive and linear element such as a partially transparent mirror [35], placed in a chain. Unlike the mechanism proposed by Sivaprakasam and coworkers [29] the emergence of zero lag and identical synchronization between the outer elements is a consequence of a true collective behavior between all the elements of the chain and cannot be explained by a combination of two different mechanisms.

Isochronous synchronization between delay-coupled lasers has recently also been found in coupled ring and chain topologies [50]. See Refs. [51,52] for a discussion of the dynamics of three semiconductor lasers arranged in a circular array.

\section{ACKNOWLEDGMENTS}

This work is supported by the MEC Spain and Feder, Projects No. TEC-2006-1009/MIC, TEC-2006-28105-E, and No. FIS2007-60327, the EC Projects PICASSO No. IST2005-34551 and GABA FP6-NEST Contract No. 043309. R.V. also acknowledges the support of the Hertie Foundation. 
[1] Y. Kuramoto, Chemical Oscillations, Waves and Turbulence (Springer, Berlin, 1984).

[2] A. Pikovsky, M. Rosenblum, and J. Kurths, Synchronization: A Universal Concept Nonlinear Science (Cambridge University Press, Cambridge, U.K., 2002).

[3] L. M. Pecora, T. L. Carroll, G. Johnson, D. Mar, and J. Heagy, Chaos 7, 520 (1997).

[4] R. Mirollo and S. Strogatz, SIAM J. Appl. Math. 50, 1645 (1990).

[5] W. Singer, Nature (London) 397, 391 (1999).

[6] L. Glass, Nature (London) 410, 277 (2001).

[7] I. Kiss, Y. Zhai, and J. Hudson, Science 296, 1676 (2002).

[8] L. M. Pecora and T. L. Carroll, Phys. Rev. Lett. 64, 821 (1990).

[9] K. Wiesenfeld, P. Colet, and S. H. Strogatz, Phys. Rev. Lett. 76, 404 (1996)

[10] G. D. VanWiggeren and R. Roy, Science 279, 1198 (1998).

[11] Z. Neda, E. Ravasz, Y. Brechet, T. Vicsek, and A. Barabasi, Nature (London) 403, 849 (2000).

[12] J. Mulet, C. Masoller, and C. R. Mirasso, Phys. Rev. A 65, 063815 (2002).

[13] T. Heil, I. Fischer, W. Elsässer, J. Mulet, and C. R. Mirasso, Phys. Rev. Lett. 86, 795 (2001).

[14] A. Takamatsu, T. Fujii, and I. Endo, Phys. Rev. Lett. 85, 2026 (2000).

[15] P. R. Roelfsema, A. K. Engel, P. König, and W. Singer, Nature (London) 385, 157 (1997).

[16] R. Vicente, L. Gollo, I. Fischer, C. R. Mirasso, and G. Pipa, Proc. Natl. Acad. Sci. U.S.A. 105, 17157 (2008).

[17] H. Schuster and P. Wagner, Prog. Theor. Phys. 81, 939 (1989).

[18] A. Hohl, A. Gavrielides, T. Erneux, and V. Kovanis, Phys. Rev. Lett. 78, 4745 (1997).

[19] H. Fujino and J. Ohtsubo, Opt. Rev. 8, 351 (2001).

[20] N. Fujiwara, Y. Takiguchi, and J. Ohtsubo, Opt. Lett. 28, 1677 (2003).

[21] J. Mulet, C. Mirasso, T. Heil, and I. Fischer, J. Opt. B: Quantum Semiclassical Opt. 6, 97 (2004).

[22] S. Tang, R. Vicente, M. Chiang, C. R. Mirasso, and J. M. Liu, IEEE J. Sel. Top. Quantum Electron. 10, 936 (2004).

[23] R. Vicente, S. Tang, J. Mulet, C. R. Mirasso, and J. M. Liu, Phys. Rev. E 70, 046216 (2004).

[24] R. Vicente, S. Tang, J. Mulet, C. R. Mirasso, and J. M. Liu, Phys. Rev. E 73, 047201 (2006).

[25] E. A. Rogers-Dakin, J. Garcia-Ojalvo, D. J. DeShazer, and R. Roy, Phys. Rev. E 73, 045201(R) (2006).

[26] J. K. White, M. Matus, and J. V. Moloney, Phys. Rev. E 65, 036229 (2002).

[27] J. Javaloyes, P. Mandel, and D. Pieroux, Phys. Rev. E 67, 036201 (2003).

[28] H. Erzgraber, D. Lenstra, B. Krauskopf, E. Wille, M. Peil, I. Fischer, and W. Elsäßer, Opt. Commun. 255, 286 (2005).
[29] S. Sivaprakasam, P. S. Spencer, P. Rees, and K. A. Shore, Opt. Lett. 28, 1397 (2003).

[30] J. Martinez Avila, R. Vicente, J. R. Rios Leite, and C. R. Mirasso, Phys. Rev. E 75, 066202 (2007).

[31] T. Yamamoto, I. Oowada, H. Yip, A. Uchida, S. Yoshimori, K. Yoshimura, J. Muramatsu, S. Goto, and P. Davis, Opt. Express 15, 3974 (2007).

[32] H. G. Winful and L. Rahman, Phys. Rev. Lett. 65, 1575 (1990).

[33] J. R. Terry, K. S. Thornburg, Jr., D. J. DeShazer, G. D. VanWiggeren, S. Zhu, P. Ashwin, and R. Roy, Phys. Rev. E 59, 4036 (1999).

[34] I. Fischer, R. Vicente, J. M. Buldu, M. Peil, C. R. Mirasso, M. C. Torrent, and J. Garcia-Ojalvo, Phys. Rev. Lett. 97, 123902 (2006).

[35] R. Vicente, I. Fischer, and C. Mirasso, Opt. Lett. 32, 403 (2007).

[36] A. Wagemakers, J. M. Buldu, and M. A. F. Sanjuan, Chaos 17, 023128 (2007).

[37] B. B. Zhou, and R. Roy, Phys. Rev. E 75, 026205 (2007).

[38] M. Peil, L. Larger, and I. Fischer, Phys. Rev. E 76, 045201(R) (2007).

[39] A. S. Landsman and I. B. Schwartz, Phys. Rev. E 75, 026201 (2007).

[40] O. D’Huys, R. Vicente, T. Erneux, J. Danckaert, and I. Fischer, Chaos 18, 037116 (2008).

[41] A. Bellen and M. Zennaro, Numerical Methods for Delay Differential Equations (Oxford University Press, Oxford, 2003).

[42] D. Lenstra, B. Verbeek, and A. Den Boef, IEEE J. Quantum Electron. 21, 674 (1985).

[43] J. Mork and B. Tromborg, IEEE J. Quantum Electron. 28, 93 (1992).

[44] M. Golubitsky and I. Stewart, The Symmetry Perspective (Birkhauser, Basel, 2003).

[45] K. M. Cuomo and A. V. Oppenheim, Phys. Rev. Lett. 71, 65 (1993).

[46] P. Colet and R. Roy, Opt. Lett. 19, 2056 (1994).

[47] C. R. Mirasso, P. Colet, and P. Garcia-Fernandez, IEEE Photonics Technol. Lett. 8, 299 (1996).

[48] I. Fischer, Y. Liu, and P. Davis, Phys. Rev. A 62, 011801(R) (2000).

[49] A. Argyris, D. Syvridis, L. Larger, V. Annovazzi-Lodi, P. Colet, I. Fischer, J. Garcia-Ojalvo, C. Mirasso, L. Pesquera, and A. Shore, Nature (London) 437, 343 (2005).

[50] G. Van der Sande, M. C. Soriano, I. Fischer, and C. R. Mirasso, Phys. Rev. E 77, 055202(R) (2008).

[51] J. Buldu, J. Garcia-Ojalvo, and M. Torrent, J. Lightwave Technol. 25, 1549 (2007).

[52] C. Gonzalez, C. Masoller, M. Torrent, and J. Garcia-Ojalvo, Europhys. Lett. 79, 64003 (2007). 